\title{
Croisements disciplinaires, enjeux didactiques : actualité d'une confrontation littérature / sciences
}

\section{Evelyne Bedoin}

\section{(2) OpenEdition}

Journals

\section{Édition électronique}

URL : http://journals.openedition.org/pratiques/1542

DOI : 10.4000/pratiques. 1542

ISSN : 2425-2042

\section{Éditeur}

Centre de recherche sur les médiations (CREM)

\section{Édition imprimée}

Date de publication : 15 juin 2010

Pagination : 161-178

\section{Référence électronique}

Evelyne Bedoin, «Croisements disciplinaires, enjeux didactiques : actualité d'une confrontation littérature / sciences », Pratiques [En ligne], 145-146 | 2010, mis en ligne le 15 juin 2010, consulté le 19 avril 2019. URL : http://journals.openedition.org/pratiques/1542 ; DOI : 10.4000/pratiques.1542 


\title{
Croisements disciplinaires, enjeux didactiques : actualité d'une confrontation littérature / sciences
}

\author{
Evelyne Bedoin
}

IUFM d'Amiens, Université de Picardie Jules-Verne

\section{Actualité d'une recherche}

L'idée de mener une étude comparative sur l'interprétation du réel dans les sciences de la nature et l'interprétation du récit de fiction a pris corps au détour de la réflexion sur la lecture littéraire, dans les années 2000. A l'instigation de Catherine Tauveron qui en a dirigé les travaux, elle a entre autres donné naissance à une thèse de doctorat que nous avons initiée sous l'intitulé « Lire le texte, lire le monde : du jeu interprétatif en littérature et en sciences ». Cette recherche, soutenue en novembre 2008 à l'Université de Rennes 2, a en effet voulu ouvrir un champ d'investigation dans l'espace des pratiques de lecture. Elle a souhaité interroger le découpage qu'effectue le monde scolaire dans l'approche de ses objets, notamment ceux du monde littéraire et ceux du monde empirique, et, par là-même, réinterroger les pratiques d'enseignement du fait littéraire. Articuler les rapports entre interprétation et objets du monde : telle a été, en effet, la préoccupation qui a guidé la recherche interdisciplinaire que nous avons poursuivie au long de notre ouvrage. Nous avons sélectionné un objet d'étude : le débat interprétatif. Du côté des pratiques enseignantes, nous nous sommes appliquée à analyser la gestion qu'un même enseignant fait du débat en littérature et en sciences.

\subsection{Interrelations disciplinaires : état de la question}

On se propose en premier lieu de mettre au jour, dans cet article, un ensemble de facteurs qui ont présidé à l'émergence d'une question de recherche interdisciplinaire autour de l'interprétation. Si des rapprochements entre deux disciplines réputées aussi incompatibles que le français et les sciences ont pu s'opérer dès la période de l' « éveil », à la fin des années 70, c'est en réalité grâce à l'action conjuguée d'infléchissements d'ordre théorique, d'évolutions didactiques et de dispositions institutionnelles. On rendra compte de quelques-uns de ces croisements.

Sous l'impulsion des Instructions Officielles de 1977 / 78, les interrelations dis- 
ciplinaires sont en effet vues, à l'ère de la linguistique communicationnelle, comme un lieu de tressage des apprentissages langagiers et scientifiques. L'on envisage les compétences linguistiques au service de l'objectif scientifique, et réciproquement. (Ducancel et Astolfi, 1995, p. 5) L'approche du réel s'articule à l'idée de communication et s'y orchestre autour de la fonction référentielle, au travers des objets du monde que sont les référents. Via la pédagogie de projet, les démarches d'approche du réel sont ainsi, dès l'origine, communes aux activités de français et aux activités scientifiques : l'éveil promeut une démarche scientifique d'analyse de la langue qui donne même statut à l'exploration du réel et à celle du langage. Les élèves sont mis face à des problèmes qui favorisent la prise d'indices, l'émission d'hypothèses, leur vérification. Emerge une démarche scientifique de résolution de problèmes concernant la langue et ses usages. Les publications INRP, qui englobent aussi bien le français que l'éveil scientifique (le terme « éveil » est significativement commun aux deux disciplines), proposent de nouvelles voies pour penser la confrontation disciplinaire. On y consigne des similitudes d'approche de faits de langue et de faits empiriques et en même temps la différence des postures d'approche du monde.

La décennie 80 est quant à elle marquée, avec l'essor des travaux de Jean-Michel Adam, par les avancées des linguistiques textuelles : l'activité scientifique est alors couplée à la lecture / écriture de textes. Les recherches en didactique s'acheminent en effet vers la caractérisation du fonctionnement des types de textes et des types d'écrits. Ce tournant marque un changement dans la démarche d'approche du réel : l'intérêt pour la typologie des textes croise la réflexion sur le modèle explicatif en sciences. Cette dernière ne s'organise plus autour des objets du monde, mais autour de la construction de ces objets, ce que l'on nomme à l'époque le « texte» du savoir. La nouveauté est de tisser les critères de scientificité avec les caractéristiques propres aux types d'écrits. Au-delà du chassé-croisé entre démarches scientifiques et typologies textuelles, on cible plus particulièrement des apprentissages scientifiques concernant le statut des savoirs et des idées, notamment la distinction entre la réalité elle-même et les idées qui sont une interprétation de la réalité (Veslin, 1988).

Les Instructions Officielles de 1995 procèdent de leur côté à un redécoupage des programmes en champs disciplinaires. Les contenus d'enseignement / apprentissage se déclinent désormais en compétences à construire chez les élèves. Les chercheurs en didactique optent à nouveau pour un ancrage des compétences dans leur discipline. Or, les didactiques du français et des sciences, dans les années 90, se rencontrent autour des pratiques sociales comme référence majeure des savoirs visés à l'école. Elles s'appuient sur le concept de pratiques sociales de référence élaboré par Jean-Louis Martinand (1986). Tel est le cas des pratiques de communication scientifique et des caractéristiques du travail des chercheurs dans le champ de la didactique des sciences.

La didactique du français interroge pour sa part les pratiques sociales de communication : l'écriture littéraire, l'oral et l'écrit dans les médias. Cette mutation dans les cohérences disciplinaires s'explique, dans le domaine des sciences du langage, par l'essor de la pragmatique du discours qui insère les conditions sociales de production des énonciateurs : on tient davantage compte des rites et des interactions qui organisent la communication. (Peterfalvi et Jacobi, 2003) Ainsi est mis en avant, sur fond de lutte institutionnelle contre l'échec scolaire, le rôle de l'argumentation orale dans la démarche scientifique. Les conduites argumentatives sont alors étudiées dans leur finalité heuristique d'établissement de la vérité.

L'intérêt pour la science « en train de se faire » apparaît comme facteur déterminant de l'émergence d'une problématique de l'interprétation dans le champ des di- 
dactiques des sciences. Cette promotion est liée au changement de statut du savoir aux différentes étapes de la démarche scientifique. Un chercheur comme Clive Sutton (1995) souligne le contraste entre deux types de langage : le langage utilisé de manière intentionnelle comme « système interprétatif», afin de générer une compréhension nouvelle, et le langage devenu "système d'étiquetage », destiné à transmettre de l'information. L'idée d'un langage interprétatif, caractéristique d'un savoir conjectural inscrit dans une réflexion sur les possibles, se répand; ces écrits intermédiaires prennent sens dans une double interaction : avec des actions sur le réel et des débats dans la classe. Parallèlement s'engage une réflexion sur le modèle scientifique comme interprétant, au sens peircien (une représentation de l'objet selon le point de vue de l'échange en cours). Le réel se distingue ainsi du perceptible : il apparaît multidimensionnel, fruit de modélisations diverses entrant dans une chaîne d'interprétants. (Weisser, 1995)

A cette époque, la recherche INRP $1^{\text {er }}$ degré « Didactisation de la lecture et de l'écriture littéraires du récit à l'école, cycles 2 et 3 » se donne pour objectifs de déterminer et d'éprouver les conditions favorables à une initiation précoce des élèves à la lecture littéraire. Le couple compréhension / interprétation, qui clive école et collège, est alors interrogé : les deux termes de la dyade ne sont plus posés, désormais, dans un rapport hiérarchique mais dialectique : l'interprétation, en littérature, devient objet d'étude à l'école primaire. Les écrits intermédiaires sont considérés comme le lieu d'émergence d'interprétations individuelles dont ils favorisent la confrontation au sein de débats interprétatifs. Ils sollicitent la singularité de la lecture individuelle et sont forgés sur le principe des écrits réflexifs en sciences.

Dans les Instructions Officielles de 2002, la discipline « français » se reconfigure. Elle comporte désormais une composante spécifique, un domaine « littérature » clairement circonscrit, et une composante transversale, la maîtrise du langage et de la langue française. Au sein des divers domaines disciplinaires, la séquence didactique s'organise autour de trois volets, « dire-lire-écrire ». Différents acquis des recherches en didactique sont intégrés. L'oral et l'écrit, s'ils se soutiennent mutuellement, se sont respectivement stabilisés dans une forme comme le débat réglé, un outil comme le carnet d'expériences en sciences et le carnet de littérature en français, témoins d'une « écriture pour soi ».

La recherche, quant à elle, dénote plusieurs tendances. Les différentes didactiques renouent il est vrai avec l'épistémologie et l'histoire de leur discipline dans une nécessaire exigence de redéfinition. En sciences, les travaux de Christian Orange (2003) s'emploient à organiser les relations entre savoirs et langages par des repères épistémologiques précis. Mais le dialogisme bakhtinien revient à l'honneur, au travers du constat qu'il n'existe de réel dialogisme que dans la mise en interaction, non des subjectivités, mais des contextes. Bronckart $(1985$, p. 8) voit lui aussi les textes comme des productions sociales et souligne les relations d'interdépendance entre les productions langagières et leur contexte d'élaboration. Dans cet intérêt pour la contextualisation des énoncés, les disciplines resserrent leurs territoires autour d'objets communs, tels les genres et les postures. Le réel se donne à lire au sein de médiations culturelles et discursives, à l'instar du récit de fiction en sciences. Une notion comme celle de posture devient centrale dans la mesure où elle invite à faire le lien entre un contexte et des pratiques langagières, où elle permet d'analyser les dysfonctionnements tant cognitifs que langagiers et de renouveler l'interprétation des différences d'apprentissage entre élèves d'une même communauté scolaire. Elle devient un outil au service de l'interdisciplinarité. 


\subsection{Convergences didactiques}

Si des disciplines comme le français et les sciences se sont retrouvées en présence, dans la longue histoire de leurs relations, autour de la maîtrise de la langue et des typologies textuelles, la décennie 90 voit arriver une identité de démarches de questionnement des objets du monde qui laisse place à l'activité interprétative. Deux paradigmes complémentaires ont joué un rôle central dans ces éléments de convergence. On en soulignera l'influence.

\subsubsection{Le paradigme de la singularité}

Il s'impose dans les sciences comme il l'a fait en littérature. On en trouve trace dans d'autres champs théoriques comme la philosophie, avec les travaux de Clément Rosset sur «l'objet singulier » (1979) et de Jean-Luc Nancy, sur le « singulier pluriel » (1996), mais aussi la linguistique avec les travaux de Catherine KerbratOrecchioni (1980). Il se signale, en didactique des sciences, par l'abandon de la démarche OHERIC ${ }^{(1)}$ pour la mise en avant de l'heuristique scientifique (la science qui se fait), outillée par les écrits personnels de recherche ou carnets d'expérience. L'enjeu est de permettre aux élèves de comprendre la nature de la science en reconnectant le savoir aux êtres humains qui l'ont établi. Discuter d'interprétations alternatives prend alors sa place dans l'institution de ce savoir. Mais la lecture singulière du monde s'inscrit comme étape de la démarche scientifique vers une écriture d'expression plus collective.

Parallèlement, la lecture littéraire se veut, comme s'accorde à le dire Bertrand Gervais (1998), « l'acte volontaire et singulier d'appropriation d'un lecteur singulier ». Il n'existe donc pas de texte littéraire, rappelle Pierre Bayard (1998, pp. 127 128), sans la subjectivité de celui qui le lit. L'intérêt se déplace alors du texte au lecteur. «C'est le lecteur qui vient achever l'œuvre et refermer le monde qu' elle ouvre, et il le fait chaque fois de manière différente. " Pour Catherine Tauveron et son équipe, l'enjeu est de « faire goûter ce plaisir particulier qui consiste à être le partenaire actif d'un jeu avec un texte qui a du jeu [...], jeu dont il convient à tout moment d'inventer les règles. » (1999, p. 12) La lecture, dès lors, n'obéit plus à un ensemble de processus automatisés comme peut l'être la compréhension, mais engage un sujet apte à interpréter l'information. Les activités de partage permettent aux élèves de confronter leur propre lecture à celle d'autrui, d'exercer la nature spéculative de l'interprétation et d'évaluer, par la métacognition, la pertinence de leurs propositions. (1999, p. 24)

\subsubsection{La pensée par problèmes}

D'autre part, la pensée par problèmes, déjà présente au moment de l'éveil dans un projet de globalisation des savoirs, s'enracine désormais dans des didactiques disciplinaires qui se sont développées chacune selon des avenues spécifiques. Elle ouvre la voie, en littérature et en sciences, à la pratique du débat comme activité de résolution (ou de construction) de problèmes ouverts. Le paradigme du problème, comme

(1) Ce schéma-type qui caractérise souvent la démarche expérimentale (Observation, Hypothèse, Expérience, Raisonnement, Interprétation, Conclusion) est en réalité une reconstruction a posteriori qui correspond davantage chez les chercheurs à une logique d'exposition de travaux que de recherche. GIORDAN, A. (2002), "C'est quoi ? Maîtresse... », Les Cahiers pédagogiques n409, «Expérimenter», pp. 22-23. 
on l'a appelé, est lié en sciences à l'épistémologie bachelardienne. Au cœur de l'activité scientifique, il permet la production de connaissances. Il est même vu, selon Popper (1991), comme le moteur de sa progression. La démarche scientifique apparaît comme une activité de résolution de problèmes, voire de problèmes ouverts : ces derniers s'avèrent plus attentifs à la construction du problème ; plusieurs procédures de résolution y sont possibles pour arriver à la solution ; les échanges entre élèves, dans une perspective initialement socioconstructiviste, y sont plus importants. (Boilevin, 2005) Construction des problèmes, argumentation, et débat scientifique dans la classe sont ainsi intimement liés.

Pour les chercheurs de l'équipe INRP « Didactisation de la lecture et de l'écriture littéraires du récit à 1'école, cycles 2 et 3 », lire littérairement un texte peut aussi être assimilé à une activité de résolution de problèmes. La lecture littéraire s'ancre en effet dans les problèmes de lecture posés par les textes. Tous les textes cependant ne présentent pas les mêmes problèmes de lecture. Deux catégories peuvent en effet être distinguées, sans dichotomie excessive : les textes lacunaires ou réticents, les textes ouverts ou proliférants. C'est la prolifération qui est source de problèmes d'interprétation : les textes proliférants offrent « de nombreux éléments potentiellement polysémiques, des indices pouvant entrer dans plusieurs réseaux et donc diversement interprétables ». (Tauveron, 1999, p. 20) Dans cette perspective, l'interprétation se fait non pas résolution de problèmes ouverts, mais « résolution ouverte de problèmes ouverts ». (Tauveron, 2002, p. 33) Par résolution ouverte, on veut signifier que le jeu interprétatif n'implique pas de solution unique à un problème posé, mais admet, comme processus de résolution, la coexistence d'une pluralité d'interprétations dans les limites de leur recevabilité. Parmi les dispositifs heuristiques (adaptés aux problèmes posés par les textes) qui permettent d'initier, au primaire, l'activité interprétative, figure le débat d'interprétation.

\subsection{Vers une nouvelle problématisation : cadres conceptuels}

Poser le problème de l'interprétation dans un contexte interdisciplinaire conduit à en renouveler la problématique. En littérature, l'interprétation a été jusque-là modélisée, sur la base de la conceptualisation de l'objet littéraire, dans ses rapports avec la compréhension. Réinterroger les frontières disciplinaires invite cette fois à poser le problème des articulations entre interprétation et référentiation, c'est-àdire entre interprétation et objets du monde. Interprète-t-on, en effet, un objet du monde physique comme on interprète un objet littéraire ? Pour répondre à cette question, nous avons cherché à articuler les notions d'interprétation et de monde en rapprochant lecture du texte et lecture du monde ; et parce que les objets disciplinaires sont façonnés par les communautés interprétatives qui les ont forgés, nous avons replacé cette étude dans le cadre de la constitution des communautés interprétatives, au sens d' " ensemble d'individus ayant intériorisé des normes et des attentes » (Fish, 1960, p. 20).

\subsubsection{Un axe fédérateur : la notion de «cadre de référence »}

Il s'avère que les théories constructivistes de Nelson Goodman (1992) trouvent un récent écho dans les travaux du Groupe de Recherche sur la Lecture (GREL) de l'Université du Québec à Montréal, groupe dont les principaux représentants sont Bertrand Gervais et Rachel Bouvet. Pour Goodman, on ne peut concevoir de réel préalable à l'activité de l'esprit : il existe une pluralité irréductible de mondes. Les mondes pluriels, toutefois, ne sont pas des mondes de substitution, exclusifs l'un de 
l'autre, mais des versions différentes du monde, parfois concurrentes. L'émergence du soleil, par exemple, peut trouver une explication dans une version de monde inscrite dans un cadre de référence géocentriste ou héliocentriste, (celui de l'expérience visuelle pour la première ou de l'astronomie pour la seconde). Or les exemples ne se limitent pas au domaine scientifique et peuvent être transférés à d'autres systèmes de description du monde comme le domaine artistique ou littéraire. Pour Goodman, chacun des systèmes construit son monde différemment.

Les théories de la lecture ont de leur côté incorporé les recherches de Goodman sur les cadres de référence. Inge Crosman Wimmers, dans sa poétique de la lecture (1988), introduit ainsi le concept de cadres de référence multiples qui permettent l'intégration de différentes perspectives critiques. Plus récemment, Rachel Bouvet (1998, p. 94) reprend cette notion à son compte dans ses travaux sur l'effet-fantastique en littérature. Un même événement, au sein du récit cette fois, peut en effet être interprété comme élément signifiant et entrer dans une version de monde, à l'intérieur de cadres de lecture différents (réaliste, allégorique...). C'est ainsi que l'épisode de pêche central, dans J'ai rêvé d'une rivière, l'album d'Allen Say ${ }^{(2)}$ que nous avons choisi pour notre expérimentation, peut être interprété comme un rêve, voire un rêve éveillé, ou une aventure initiatique, éventuellement magique. Par magie, on entend cette traversée des mondes qui fait que l'escapade faite à la brune prend des accents de réalité. Chacune de ces versions de monde prend place dans un cadre référentiel différent : psychobiologique pour le rêve, surnaturel ou merveilleux pour l'expérience d'« éveil » qui se vit sur la rivière comme réalisation et abandon d'un désir de pêche, cadre idéologique, encore, si l'on considère la préservation de l'équilibre naturel comme le rêve des hommes, leur Utopie. L'effet fantastique repose sur l'indétermination de ces cadres de référence, indétermination dont joue précisément l'album d'Allen Say.

Les cadres référentiels se présentent ainsi comme des grilles de lecture du monde. Jean-Louis Dufays, dans son approche de la stéréotypie (1994, p. 100), les classe dans les ensembles de codes, systèmes de références ou systèmes interprétatifs institutionnalisés (codes d'inventio) dont dispose le lecteur. Ils ressortissent à ce que 1'on peut appeler par ailleurs des "écoles de pensée ». Christian Orange (1997, pp. 46-50) développe en sciences, à l'occasion de ses travaux sur la modélisation, la notion convergente de registre explicatif. Le concept entretient des affinités avec celui de paradigme ; mais au lieu de correspondre à l'ensemble des références théoriques d'un domaine, il n'en constitue qu'un soubassement explicatif (de type géocentriste par exemple) et peut être individuel.

\subsubsection{Une identité définitionnelle}

Interpréter, dans un contexte littéraire de multiréférentialité, consiste à lever les ambiguïtés, à placer le texte dans telle ou telle perspective pour en reconstruire la cohérence. L'activité demande ainsi aux élèves une opération de clichage : faire entrer une version d'événement dans un cadre référentiel spécifique (Dufays 1994, p. 158). Cette interprétation-là se place bien sur le « terrain du jeu libre de l'intelligence et de la subjectivité » (Vandendorpe, 1992, p. 160). Parallèlement, dans la modélisation scientifique, l'interprétation correspond à la sélection des variables, mais engage le registre explicatif qui la gouverne. Alain Vergnioux, philosophe de l'éducation (2003, p. 28), rappelle avec Gadamer les liens qui unissent compréhen-

(2) L'album, sorti en 2001, est édité à l'Ecole des loisirs. 
sion et interprétation pour offrir quelques éléments définitoires. Interpréter, «c'est comprendre, [en effet], de façon différentielle », en faisant « le choix de telle ou telle perspective, de tel ou tel modèle théorique ».

\subsection{Objectifs disciplinaires et interdisciplinaires}

Nous avons ainsi en vue la constitution de communautés interprétatives scolaires. Elles se situent dans le cadre plus général des communautés discursives, cadre permettant de cerner et de transposer les conditions sociales et culturelles d'élaboration des savoirs (Bernié, 2002, p. 78). Chaque discipline comporte ses propres pratiques langagières et ses propres genres discursifs. Nous considérons à vrai dire ces derniers comme formes didactiques plus que comme genres extrascolaires. C'est sous cet angle que nous avons sélectionné comme objets d'étude, pour les confronter, le débat intégré à la discipline littéraire et à la discipline scientifique. Ils présentent en commun la caractéristique d'être des espaces intersubjectifs et un lieu de secondarisation des pratiques langagières. Précisons que l'attitude de secondarisation consiste à faire des objets du monde un monde d'objets à interroger, sur lesquels exercer des opérations de pensée et un travail intellectif(Bautier et Goigoux, 2004, p. 91). La construction de systèmes interprétatifs, comme la pratique de la modélisation, participe de cette activité de secondarisation.

Dans ce contexte d'institution de communautés interprétatives scolaires, nous avons tenté de mesurer l'impact du jeu interprétatif, au sein du débat, sur la construction de postures disciplinaires comme rapport au monde et modes d'entrée dans ces communautés. Construire ce rapport consiste à s'approprier, afin de penser le monde, les savoirs, les démarches, les règles de validité spécifiques à chaque discipline. En ce sens, l'accent a été mis plus particulièrement sur le paramètre « cadre de référence ». Nous y avons vu un point d'ancrage dans les postures disciplinaires. L'analyse s'est ainsi centrée sur la manière dont les interprétations s'ancrent dans des systèmes d'intelligibilité et dont le débat déplace ces cadres de référence. L'enjeu en a été de trouver des points d'appui pour permettre à l'élève de passer d'un monde à l'autre, de réorganiser sa « lecture du livre du monde », ou bien encore de favoriser la circulation des savoirs d'un monde disciplinaire à un autre. Du côté de la pratique enseignante, notre préoccupation s'est portée sur la manière dont le mâ̂tre accompagne ce changement de posture ; pour ce faire, nous avons interrogé la propre posture de l'enseignant en fonction d'une variable liée à l'interdisciplinarité, le changement de contexte discursif.

\section{Jeux de cadrage et gestes enseignants}

\subsection{Choix méthodologiques}

C'est donc un double objectif de mise en regard disciplinaire qu' a poursuivi la recherche : délimiter en premier lieu les points de convergence et de divergence qui peuvent s'établir entre débats interprétatifs littéraires et scientifiques, en classe de cycle 3 ; en second lieu, regarder du point de vue des pratiques enseignantes et proposer des lieux de réflexion, pour la formation, dans la gestion de contextes discursifs différents. Posture du maître et posture de l'élève ont ainsi été confrontées dans l'idée de déterminer des types de rupture susceptibles d'entraver la constitution d'une communauté interprétative et de déclencher la réflexion.

Pour cette raison, nous avons fait appel à un corpus élargi permettant de croiser 
les points de vue des acteurs de la situation scolaire sur un même objet d'étude. L'observation du travail de l'élève dans la classe, en petit ou en grand groupe, a donc été complétée par des entretiens d'auto-confrontation plaçant le maître face aux enregistrements des débats mis en place dans les deux disciplines, littéraire et scientifique : l'enseignant y analyse sa pratique en présence du formateur-chercheur. Le protocole d'entretien d'auto-confrontation est emprunté à la méthodologie utilisée en psychologie ergonomique. L'intérêt est de faire émerger la posture épistémologique du maître et de comparer visée du maître et visée du chercheur dans l'analyse des données.

L'expérimentation a été menée en 2004 / 2005 dans une classe rurale de l'Aisne et porte sur quelques études de cas. L'enseignante devait gérer une classe à double niveau, CM1 / CM2. Le protocole d'expérimentation a misé sur une homologie de situations d'activités de résolution de problèmes en littérature et en sciences. Il a essentiellement rapproché, en effet, deux problèmes d'ordre explicatif, l'explication du phénomène de l'éruption volcanique en sciences et l'explication du rêve de Marc, le héros de J'ai rêvé d'une rivière.

\subsection{Gestes enseignants et contextes discursifs}

Dans le cadre de cet article, et pour souligner quelques éléments convergents avec de récentes recherches en didactique, nous ciblerons l'analyse sur l'étude de gestes enseignants. Celle-ci se place dans la perspective de la gestion d'un contexte discursif qui incorpore, de facto, le paramètre «cadre de référence ». En effet, l'explication du phénomène éruptif en sciences de la terre varie selon les contextes historiques, de l'existence d'un feu central au modèle de la combustion, en passant par la conception de la «montagne creuse ». Ces explications sont désormais scientifiquement caduques, mais susceptibles de se retrouver dans les conceptions préalables des élèves (ou modèles spontanés) et peuvent aider à les catégoriser (Laperrière-Tacussel, 1995). Elles constituent souvent les « registres explicatifs » dans lesquels s'inscrivent les interprétations des élèves, propositions recueillies dans le cadre de notre expérimentation sous forme de dessins ou de schémas tenant lieu de modèle, cette « version de monde » permettant d'expliquer l'événement éruptif.

En littérature, c'est la nature du rêve de Marc, l'enfant fiévreux, qui prête comme nous l'avons vu à interrogation. L'album fait à ce propos miroiter différentes acceptions du terme, acceptions qui relèvent de registres explicatifs divers.

\subsection{1. Éléments de contextualisation}

En sciences, le phénomène de l'éruption volcanique trouve au sein de notre expérimentation un approfondissement dans une nouvelle problématique : "Pourquoi y a-t-il des volcans éruptifs et des volcans calmes (effusifs) ? La question du débat scientifique s'inscrit dans un registre explicatif déjà construit par la classe : elle s'appuie sur des savoirs admis par le groupe, comme le rôle des gaz dans l'éruption, mais vise la construction de nouveaux savoirs. L'importance relative de l'éruption est due à deux facteurs : la quantité de gaz et la viscosité du magma. En effet, lorsque le magma est très fluide, les gaz s'échappent facilement sans qu'il y ait pratiquement d'activité explosive : les volcans sont dits effusifs. En revanche, lorsque le magma est visqueux, les gaz ne peuvent s'échapper que si une très forte pression gazeuse est atteinte : il y a alors explosion, et les volcans sont dits éruptifs. Précédemment, dans la séquence, les groupes ont fait le deuil de conceptions d'ordre animiste ou artifi- 
cialiste. Les élèves proposent cette fois, pour répondre, deux schémas couplés : l'un pour l'éruption de type effusif, l'autre pour l'éruption explosive.

En littérature, l'album d'Allen Say vient clore un réseau sur les récits de rêve dont la classe a implicitement conceptualisé les caractéristiques. La découverte du texte débouche sur une question de débat : «Est-ce un rêve? ». Les élèves hésitent entre univers onirique et univers magique : lorsque Marc, alité sous l'effet de la fièvre, ouvre la boîte de leurres de pêche envoyés par son oncle Edouard, un paysage de rivière a remplacé les immeubles du quartier. La boîte est-elle magique ou a-t-elle ouvert les portes du songe ? Détail troublant, qui fait hésiter le lecteur : la boîte d'éphémères, ouverte au début de l'épisode, est fermée à la fin. En petits groupes et à l'aide d'écrits transitoires, les élèves, en formation restreinte, proposent une interprétation argumentée de l'aventure du héros, de même qu'en sciences ils présentent un modèle sous forme de schéma. Dans les deux disciplines, les groupes sont constitués à l'identique. Des voix dissidentes peuvent se faire entendre au sein des petits groupes, ce qui est le cas dans les deux fragments présentés ci-dessous.

\subsubsection{Analyse comparée d'un geste de décontextualisation / recontextualisation}

Le geste à l'étude est celui par lequel le maître accompagne l'inscription des élèves dans une communauté interprétative, autrement dit le passage d'une communauté d'origine à une communauté de destination. Il est considéré dans sa dimension langagière, en tant que conduite discursive. Tout en poursuivant notre propre objectif d'étude de l'étayage enseignant (Bruner, 1983) dans le contexte des communautés interprétatives, nous nous rapprochons ici de la notion de « geste professionnel » élaborée par Anne Jorro, Roland Goigoux et Jean-Paul Bernié pour désigner « ce qui serait un juste milieu entre généralité et précision de l'analyse, et entre transversalité et spécificité disciplinaire » (Chabannes et alii, 2008, p. 39). L'objet d'enseignement, en tant qu'objet à construire sous forme de possible littéraire ou scientifique, tient en effet une place centrale au cœur du débat, comme l'a mis en évidence le « modèle de l'agir enseignant et de son ajustement» (Bucheton, 2008), et c'est bien ce qui oriente les interventions magistrales sans être pour autant le seul facteur en cause. Nous soulignerons la dimension pragmatique de cet agir du maître.

Dans les deux extraits mis en parallèle, l'enseignante $(M)$ tente une opération de décadrage pour susciter un réajustement de la part de l'élève et un changement de posture. Ce décadrage passe par la déconstruction du cadre de référence magique, cadre dans lequel s'inscrit l'interprétation envisagée. C'est un geste que l'enseignante réitère d'un débat à l'autre, aussi bien dans un contexte scientifique que dans un contexte littéraire. Il ne sera pas tenu compte ici, précisons-le, des recherches d'impossibilités émises en sciences par les élèves d'autres groupes dans la classe, ces impossibilités qui favorisent l'articulation entre faits et idées, ou sur les arguments émis par les pairs en classe de littérature.

\section{a) fragments de débats : déconstruction du registre magique}

Dans l'un et l'autre cas, l'enseignante met en œuvre une conduite argumentative à usage pragmatique : la visée, déconstructrice, ambitionne le positionnement des acteurs de l'échange au sein d'une communauté interprétative. Mais le geste ne revêt pas les mêmes formes d'un débat à l'autre. Nous attribuons cet écart à une différence de gestion de l'espace tensionnel (lié à l'objet) qui s'exerce entre cadres de référence au sein du débat interprétatif littéraire et du débat scientifique, ce qu'une analyse plus fine va préciser. 


\begin{tabular}{|c|c|}
\hline Débat scientifique & Débat littéraire \\
\hline $\begin{array}{l}305 \text { Max - maîtresse ça serait avec la } \\
\text { lave maîtresse / à un moment a're- } \\
\text { coulerait/ maîtresse // sur les côtés } \\
\text { maîtresse (pointe le schéma du vol- } \\
\text { can effusif) } \\
306 \text { M-alors d'un coup on est dans un } \\
\text { film de magie / la lave se resserre } \\
\text { pour laisser passer l'gaz // et hop il } \\
\text { y en a assez / la lave se remet contre } \\
\text { les parois / voilà bienvenue au } \\
\text { monde merveilleux de la fée } \\
\text { Maxence // est-ce que ça tient de- } \\
\text { bout ce que tu dis? }\end{array}$ & $\begin{array}{l}146 \begin{array}{l}\text { M-quel est pour vous l'indice / An- } \\
\text { toine / qui montrerait que c'est de la } \\
\text { magie? }\end{array} \\
147 \begin{array}{l}\text { Ant - ben à la fin quand il revient } \\
\text { chez lui eh ben toutes les lumières } \\
\text { elles sont allumées sauf sa chambre }\end{array} \\
148 \begin{array}{l}\text { M - et alors ? eh ben oui pasque } \\
\text { pour vous il dort / et pour lui il dort } \\
\text { pas / et alors c'est magique? }\end{array} \\
149 \text { Ant - ben oui maîtresse } \\
150 \begin{array}{l}\text { M - quand ta chambre elle est } \\
\text { éteinte / c'est magique? }\end{array}\end{array}$ \\
\hline
\end{tabular}

\section{- en sciences : une déconstruction par l'ironie}

Maxence propose, en marge de son groupe, un modèle spécifique pour l'éruption effusive : celui des deux couloirs de gaz (voir annexe). Ce schéma présente la particularité de dissocier les gaz de la lave. L'éruption effusive s'explique ainsi par la diffusion des gaz sur les côtés : ils entraînent la lave liquide qui se met à couler; sur les parois de la cheminée se forment alors comme deux couloirs de gaz, un gaz qui remonte, sur le schéma, le long des parois de la bouteille. La légende indique : " C'est une éruption calme car le gaz passe sur les côtes. ».

Le facteur causal invoqué par l'élève (305), dans la mise en commun, relève de l'aléatoire et de l'arbitraire ( s'opposent à la logique du raisonnement scientifique qui serait attendu dans la communauté de référence. L'élève conçoit en effet ses explications dans un registre anthropomorphique : la lave personnifiée se dote d'intentions animistes. Le cadre anthropomorphique entre ici ouvertement en conflit avec le registre scientifique. C'est ce cadre inadapté qui prête le flanc à la critique de l'enseignante (306) et en devient la cible. L'ironie dont elle fait preuve apparaît comme un moyen de mettre à distance les normes de pensée propres à une «école » définie : le procédé est secondarisé. On considère en effet l'ironie, à la suite de Ducrot (1984, p. 211), comme un phénomène de dédoublement de l'instance énonciative : «parler de façon ironique, cela revient, pour un locuteur $\mathrm{L}$, à présenter l'énonciation comme exprimant la position d'un énonciateur E, position dont on sait par ailleurs que le locuteur L n'en prend pas la responsabilité et, bien plus, qu'il la tient pour absurde ». Cette volonté de distanciation s'opère dans l'extrait choisi au moyen de procédés divers qui participent d'une sorte de rhétorique à usage pragmatique :

- la scène en forme d'hypotypose, quasi cinématographique (un «film»), non seulement glose l'explication de Maxence mais la détourne de manière parodique. Le coup de baguette magique renvoie au truchement d'une force secrète qui s'exercerait sur la lave en animant la matière. En poussant le modèle à la ca- 
ricature, l'intervention magistrale dénonce la manière dont l'élève fictionnalise le réel, faisant de la lave un actant doté d'intentions. Elle ôte ainsi toute validité au modèle de Maxence dans le monde physique ;

- l'enseignante recourt aux formules stéréotypées, véritables citations aisément repérables car elles constituent des références partagées entre le maître et les élèves (306 " bienvenue au monde merveilleux de la fée Maxence »). Ce faisant, elle se désolidarise de ses propos et met en scène une double voix, celle de l'acteur d'une communauté scolaire qui adhère à un cadre " magique », voire à une pensée magique (la "fée Maxence » désigne ainsi le positionnement de l'élève au compte de qui sont renvoyés les propos), et la voix d'une communauté de référence qui, elle, pense dans des standards rationnels. Cette deuxième voix se signale par l'emploi de quelques marqueurs discursifs ( « alors » " d'un coup » / " et hop » / " voilà ») qui, en soulignant l'irrationalité de l'explication, montrent que son auteur ne revendique pas ce type de positionnement, et de surcroît le conteste.

\section{- en littérature : une déconstruction par l'absurde}

Antoine avance quant à lui, dans le débat littéraire, un argument qui accrédite la version surnaturelle : la lumière éteinte. Du point de vue de l'élève, Marc, le héros de l'histoire, a réellement quitté sa demeure et se retrouve physiquement sur la rivière. C'est pourquoi il ne peut être présent dans sa chambre, qui n'est pas allumée. L'enseignante s'essaie une fois encore à déconsidérer la piste du surnaturel. Divers procédés sont ici conjugués, qui revêtent approximativement la forme d'un raisonnement par l'absurde ; ils confinent à une stratégie de ruse employée pour déjouer le supposé assujettissement de l'élève au registre magique :

- la juxtaposition des points de vue (148) est un moyen déjà utilisé par l'enseignante au cours du débat pour mettre en tension des versions d'événement qui entrent dans des cadres référentiels divers, rêve et magie. Il permet ici de poser une proposition, celle du héros éveillé, ou du moins de la faire coexister avec d'autres ;

- la contradiction logique est un biais pour ruiner l'argumentation de l'élève. A cette fin, l'enseignante recourt à un argument tiré de l'univers du quotidien et faisant appel à l'expérience commune (150). Elle pointe ainsi le décalage qui existe entre un fait banal, inscrit dans des usages de la vie courante, et la portée surnaturelle que lui confère l'élève. Elle fait alors ressortir une dissonance entre deux cadres référentiels qui se contrarient l'un l'autre, et sollicite un repositionnement de l'élève dans un univers de référence.

\section{b) deux gestes, une même implication?}

Le geste de décontextualisation / recontextualisation effectué par l'enseignante présente des caractéristiques communes d'un débat à l'autre sans pour autant revêtir les mêmes formes. En effet, il s'agit tout d'abord d'une opération quasi-similaire de négation : les interventions magistrales nient la validité du système d'intelligibilité employé par l'élève. On note bien, dans les deux cas (306/150), une adresse personnelle au récepteur qui l'implique dans la communauté scolaire. Déceler la faille, en montrer l'absurdité, telle semble être la stratégie magistrale. Mais dans l'exemple scientifique, l'enseignante montre en quoi l'anomalie de l'intentionnalité de la lave déplace le cadre de référence, du registre scientifique au registre magi- 
que; dans l'exemple littéraire, elle déplace l'indice apporté par Antoine du monde du texte au monde quotidien, afin de provoquer un choc de cadres : réaliste, magique. Elle tente, dans des contextes disciplinaires différents, de faire passer l'élève d'un cadre à un autre.

Pourtant, la négation de la valeur de vérité de la proposition émise n'a pas, en soi, la même incidence dans les deux cas. Une formule évaluative, "ça tient pas debout » signale, dans la communauté scolaire scientifique, la non-pertinence du cadre référentiel sous-jacent à l'hypothèse interprétative. Les registres explicatifs sont exclusifs l'un de l'autre : la rationalité ne peut coexister avec un monde régi par le surnaturel magique. L'enseignante met en scène ce dédoublement des cadres par l'usage de l'ironie.

On peut dire que l'attitude du maître participe d'une critique des solutions qui permet de construire une posture scientifique. Critiquer permet en effet de comprendre ce qui, parmi les hypothèses envisagées, est susceptible d'être retenu comme un «possible», en fonction de conditions de validité. C'est une des caractéristiques de l'apodictique (Orange, 2003) : la pensée se situe au centre d'un faisceau de normes qui fait qu'elle ne peut pas être autre. Ces normes sont liées au registre de référence de la classe. Progressivement d'ailleurs, au cours du débat, et à l'aide d'une confrontation entre les deux schémas dont l'un est valide, celui de l'éruption explosive, l'enseignante conduira Maxence, avec l'aide de ses pairs, à une recherche de raisons qui passent par une des propriétés du liquide : épouser la forme du contenant. Ces raisons visent à poursuivre la déconstruction du registre "magique », et à construire en revanche les normes attendues. La prise de position de l'enseignante est ici catégorique. Simultanément est ménagé, au sein de la communauté interprétative et toujours par le biais de l'ironie, un espace de négociation, autrement dit une discussion et un repositionnement possibles.

A l'inverse, la réfutation du cadre magique, à ce stade du débat littéraire, ne peut être complète et ne constitue pas une négation totale. Elle est recherche de contradictions. Toutefois, il est significatif que le système interprétatif d'Antoine ne puisse être totalement révoqué : il est fonction du point de vue que l'on adopte sur le monde représenté. La parole magistrale fait coexister ces points de vue : si l'on voit la scène les yeux ouverts, l'aventure prend la dimension du vécu; si on la voit les yeux fermés, il s'agit bien d'un rêve. L'intervention de l'enseignante apparaît plus comme une mise en soupçon que comme une annihilation. Elle est moins catégorique, et ne met pas en scène de changement de posture, comme en sciences. Les mondes évoqués s'avèrent compossibles ; c'est alors la plausibilité de l'hypothèse magique qui est mise à mal. L'objection vise plutôt une contre-argumentation de l'élève mis en demeure de prendre la défense de sa propre version de monde. L'espace de négociation se fait plus ouvert. Par la suite, en s'appuyant sur l'isotopie du merveilleux qui parcourt le texte et fait de la nature un objet tissé d'imaginaire, la classe acceptera l'irruption de la magie en tant que possible, comme registre d'écriture plutôt que comme cadre. C'est ce qu'elle conceptualise sous forme d'oxymore : « un rêve magique ». Le possible littéraire, en effet, n'obéit pas aux mêmes normes de validation que le possible scientifique.

En somme, le geste de déconstruction opéré par l'enseignante met en œuvre une conduite argumentative de réfutation dont la nature change en fonction de la variable contextuelle. Pourtant la vocation de ce geste enseignant est bien d'orienter l'espace intersubjectif dont on peut souligner qu'il ne consiste pas, d'une communauté à l'autre, à s' efforcer d'obtenir un consensus de la part des élèves; si, en sciences de la terre, il contribue à forger un point de vue homogène sur le phénomène volcani- 
que, il tend plutôt en classe de littérature à homogénéiser les points de vue sur l'événement narratif.

\subsubsection{Intentions magistrales et changement de posture}

\section{a) Rupture épistémologique en littérature}

L'entretien d'auto-confrontation invite l'enseignante à associer des intentions à ses interventions. Le formateur-chercheur s'attache, dans cet entretien, à obtenir des modalités de guidage de l'activité collective d'interprétation, à mettre au jour quelques médiations vers la construction de postures disciplinaires voire d'envisager des ruptures. Le passage sélectionné, retranscrit ci-dessous, fait retour sur le débat interprétatif littéraire, et plus particulièrement sur la proposition d'Antoine ; il questionne l'intervention de l'enseignante destinée à répudier la magie.

8 FC - mais un rêve un peu particulier / je n'sais pas / peut-être un peu magique

$9 \quad M$-certes mais la magie / ça veut rien dire la magie / il n'y a rien de magique quand c'est un rêve / tout s'explique

10 FC - c'est intéressant ce que vous dites

11 M-en plus / c'est vrai / ça n'existe pas la magie / tout s'explique / il y a quelque chose derrière / donc làj 'voulais pas pasque bon la magie / c'est aussi la facilitél bon c'est magique comme avec bon allez c'est magique / allez on n'explique rien / on laisse tomber c'est / tandis que le rêve après / on pouvait aller vers le rêve éveillé / le rêve / alors que la magie on peut pas gratter / c'est magique point bon c'est magique c'est super / tandis que le rêve derrière on peut aller chercher / "je dors » ou alors "t'es en train d'rêver» / hop « je rêve d'avoir une super maison" / des trucs comme ça / donc après on doit pouvoir gratter / et puis on peut aller plus philosophiquement chercher des trucs / tandis qu'à la limite ils m'auraient tous dit " c'est d'la magie » ben j'ai plus rien à dire //ils m'auraient prouvé qu'c'est d'la magie ben j'aurais essayé de démonter en amenant le rêve / et puis de toute façon ils m'l'ont démonté en allant rechercher

Nous avons noté jusque-là une différence de positionnement de l'enseignante lié aux modes de tension qui s'exercent entre cadres de référence d'une discipline à l'autre. Or il s'avère que l'entretien présente un décalage entre le geste de déconstruction mis en œuvre dans le débat interprétatif littéraire et l'intention qui le gouverne. L'enseignante procède en littérature à un choix de perspective qui correspond à une préférence, voire un choix de valeurs : le rêve contre la magie. Certes, elle suit en cela la piste la plus féconde, mais en attribuant à la magie le rôle de registre explicatif qu'il peut jouer en sciences (11 "çan'existe pas la magie / tout s'expli$q u e »)$ : le cadre magique est rejeté au nom de la rationalité. L'entretien tend à rabattre le monde fictionnel sur le monde réel, un réel ordonné par la raison. Or l'univers de l'album obéit à des règles génériques qui l'autorisent à une démultiplication des cadres de référence. Un même objet du monde (telle la sirène) peut appartenir à deux mondes différents, rêve et magie, et les indices se font dès lors réversibles.

Le merveilleux au sens large apparaît donc, dans J'ai rêvé d'une rivière, comme un monde possible. Mais rêve et magie ne trouvent pas ici d'articulation. Or, même si on ne peut noter d'incidence significative sur la posture de l'élève dans l'économie du débat, force est de reconnaître que le risque est du côté de la conceptualisa- 
tion que les élèves font de l'objet littéraire, et plus spécifiquement du genre fantastique. Sont en jeu également, outre les normes de validation propres à une communauté interprétative, les savoirs disciplinaires du maître et son rapport à l'épistémologie. C'est donc une rupture épistémologique que dévoile l'entretien, et que le débat seul n'aurait pu mettre au jour. Nous voyons donc un intérêt à développer cette dimension réflexive dans les pratiques de formation pour l'analyse de la gestion d'un contexte discursif.

\section{b) Savoirs et posture du maître}

Les attentes des communautés interprétatives scolaires permettent en effet de considérer sous un autre angle le rôle du maître dans la gestion du débat. Parce que cela ne va pas de soi pour des enseignants polyvalents, et que ces postures se construisent majoritairement dans l'histoire du sujet, l'enseignante de notre dispositif d'expérimentation, dont les compétences professionnelles ne sont pas à remettre en cause, semble avoir superposé ici les contextes dans le passage d'un débat à l'autre. Or la gestion des cadres de référence n'est pas similaire dans les deux disciplines : cadres incommensurables du côté scientifique, cadres compossibles du côté littéraire, dans les limites des devoirs imposés par le texte.

Cette étude de cas pose la question du décalage entre savoirs du maître et savoirs du formateur dans le passage d'une discipline à l'autre. La composante épistémologique est ainsi à prendre en considération dans la flexibilité des postures chez l'enseignant. La méconnaissance de savoirs et de postures disciplinaires peut en effet malencontreusement être source de rupture dans la constitution de communautés interprétatives. Nous dérivons ainsi pour finir de cette analyse quelques types de savoirs à interroger, à réactualiser ou à installer, chez les enseignants, dans le domaine littéraire. La liste serait à compléter :

\section{- des savoirs concernant l'objet littéraire}

La non-reconnaissance du genre, tout autant qu'une conception datée de ce genre, comme c'est le cas souvent pour le fantastique à l'école primaire où prévaut la définition de Caillois, peut être un frein à l'établissement d'une communauté interprétative littéraire. ;

\section{- des savoirs en matière de poétique}

La ligne de partage entre sens et valeurs dans un texte, la place des valeurs dans le débat interprétatif peut également être une entrave. Une réflexion sur la fabrique des valeurs dans un texte (on songe ici à Poétique des valeurs de Vincent Jouve, 2001) pourrait être un élément de clarification ;

\section{- des savoirs sur les théories et les pratiques de la lecture littéraire}

Le champ des didactiques du littéraire s'est élargi ces dernières années, au travers, notamment, des différentes recherches INRP. Existe-t-il une didactique de référence ? Remettre en perspective ces didactiques, évaluer leur contexte de pertinence, les objectifs qu'elles poursuivent et les dispositifs qu'elles promeuvent (du débat interprétatif à la conversation) donnerait plus de lisibilité à la pratique enseignante. 


\section{Quelle interdisciplinarité ?}

La complexité du champ didactique est telle de nos jours qu'il ne suffit pas de parler d'interdisciplinarité pour situer cette recherche. Relève-t-elle en effet d'une didactique interdisciplinaire, d'une interdidactique, ou d'une didactique comparée ? La première approche travaille les compétences interdisciplinaires, en particulier au sein de textes littéraires (Les derniers jours de Pompéi, d'Edward Bulwer-Lytton, pour le phénomène volcanique); les notions de posture et de genre y sont centrales. Mais elle le fait au point où les savoirs font système et n'offre pas de réelle confrontation de pratiques ; en cela notre recherche entretient davantage de liens avec la didactique comparée, dans la mesure où, avec Jean-Paul Bernié (2002), elle pose la nécessité de s'intéresser à la notion de communauté discursive. Les pratiques langagières varient en effet d'une discipline à l'autre et prêtent à confrontation. Ainsi peut s'ériger la prise de conscience de similitudes et de différences dans les pratiques, de lieux d'échanges et de transferts possibles. Ce sont les discours didactiques qui sont alors mis en regard.

Cette recherche s'est ainsi édifiée en rapport avec les didactiques de référence dans le champ disciplinaire, en particulier les travaux de Catherine Tauveron sur la lecture littéraire et ceux de Christian Orange en sciences de la nature. Elle y emprunte ses concepts. Mais elle entre aussi en tension avec ces disciplines dans la mesure où les didactiques se sont mutuellement éclairées. On retiendra juste ici quelques lieux d'échanges : l'idée de cadres de lecture, héritée de l'approche scientifique, a permis de déplacer la problématique de l'interprétation en littérature vers l'étude des systèmes d'intelligibilité et de renouer avec les avancées de Jean-Louis Dufays autour des codes d'elocutio. La modélisation didactique de l'interprétation en didactique du littéraire a fourni une référence pour cerner les contours de la notion d'interprétation en sciences, et a trouvé une caution chez Alain Vergnioux. La confrontation a permis de cibler une classe d'objets, en sciences comme en littérature : des phénomènes dotés d'une épaisseur historique (faits géologiques ou astronomiques), des textes littéraires caractérisés par l'intertextualité ou l'ambiguïté référentielle.

Ces travaux participent enfin d'un mouvement de redéfinition des disciplines scolaires : non seulement est-ce dans les croisements didactiques, mais aussi dans la manière dont ces disciplines s'enseignent, au cœur d'un espace intersubjectif, qu'elles se réactualisent. 


\section{Références bibliographiques}

BAutier, E., Goigoux, R. (2004) : « Difficultés d'apprentissage, processus de secondarisation et pratiques enseignantes : une hypothèse relationnelle », $R e^{-}$ vue française de pédagogie, $\mathrm{n}^{\circ} 148$.

BAyARD, P. (1998) : Qui a tué Roger Ackroyd?, Paris, Les Editions de Minuit.

BERNIE, J.-P. (2002) : «L'approche des pratiques langagières scolaires à travers la notion de « communauté discursive » : un apport à la didactique comparée ? », in Revue française de pédagogie $\mathrm{n}^{\circ} 141$, pp. 77-88.

BOILEVIN, J.-M. (2005) : «Enseigner la physique par situation problème ou par problème ouvert », in Aster $\mathrm{n}^{\circ} 40$, « Problème et problématisation », INRP.

Bouvet, R. (1998) : Etranges récits, étranges lectures, Essai sur l'effet fantastique, Montréal, Balzac-Le Gros Eds., L'Univers des discours.

BRONCKART, J.-P. (1985) : Le Fonctionnement des discours, Neuchâtel, Delachaux et Niestlé.

BRUNER, J., (1983) : Le développement de l'enfant : savoir faire, savoir dire, Paris, PUF.

BuCHETON, D. (dir.) (2008) : L'agir enseignant: des gestes professionnels ajustés. Publication des travaux de l'Equipe de recherche technologique en éducation (ERTé 40), « Conditions et difficultés d'entrée dans les situations d'apprentissage : les langages, vecteurs de la construction des savoirs ", 2004-2006, IUFM de Montpellier, Toulouse, Octares.

Chabannes, J.-Ch., Desault, M., Dupuy, C. et A igoin C. (2008) : « Les gestes professionnels spécifiques dans le débat interprétatif : problèmes pour l'analyse et la formation », in Repères 37, "Pratiques effectives de la littérature à l'école et au collège », p. 197-226.

Crosman Wimmers, I. (1988) : Poetics of Reading, Approaches to the Novel, Princeton, Princeton University Press, 1988.

DUCANCEL, G. et A STOLFI, J.-P. (1995) : «Apprentissages langagiers, apprentissages scientifiques, Problématiques didactiques : regards en arrière et aspects actuels », Repères 12, Paris, INRP, Didactiques des disciplines, pp. 5-20.

Ducrot, O. (1984) : Le dire et le dit, Paris, Ed. Minuit.

DuFAYS, J.-L. (1994) : Stéréotype et lecture, Liège, Mardaga.

FISH, S. (1960) : Quand lire, c'est faire, L'autorité des communautés interprétatives, Préface de Yves Citton, 2007 pour la traduction française, Paris, Les prairies ordinaires.

GERVAIS, B. (1998) : Lecture littéraire et explorations en littérature américaine, Montréal, XYZ Editeurs, coll. "Théorie et littérature ».

Goodman, N. (1992) : Manières de faire des mondes, Nîmes, Jacqueline Chambon.

Jouve, V. (2001) : Poétique des valeurs, Presses Universitaires de France, coll. « Ecriture ».

Kerbrat-Orecchioni, C. (1980) : L'énonciation. De la subjectivité dans le langage, Paris, Armand Colin.

LAPERRIERE-TACUSSEI, M. (1995) : « Le volcanisme, du cours moyen à l'IUFM », in Aster $\mathrm{n}^{\circ} 20$, «Représentations et obstacles en géologie », Paris, INRP, pp. 61-84. 
MARTINAND, J.-L. (1986) : Comprendre et transformer la matière, Berne, Peter Lang.

NANCY, J.-L. (1996) : Etre singulier pluriel, Paris, Galilée.

ORANGE, C. (1997) : Problèmes et modélisation en biologie, Quels apprentissages pour le lycée ?, Paris, PUF, coll. «L'Educateur».

- (2003) : «Débat scientifique dans la classe, problématisation et argumentation : le cas d'un débat sur la nutrition au cours moyen », in Aster n³7, Interactions langagières, Paris, INRP, pp. 83-108.

PETERFAlVI, B. et JACOBI, D. (2003) : «Les interactions langagières, entre processus et matériaux pour la recherche », Aster 37, «Interactions langagières 1 », Paris, INRP, Didactique des disciplines, pp. 3-16.

POPPER, K. (1991) : La connaissance objective, Paris, Aubier.

Rosset, C. (1979) : L'objet singulier, Paris, Les Editions de minuit, coll. « critique $»$.

SutTon, C. (1995) : «Quelques questions sur l'écriture et la science : une vue personnelle d'Outre-Manche », Repères n ${ }^{\circ}$, Paris, INRP, pp. 37-52.

TAUVERON, C. (1999) : «Comprendre et interpréter le littéraire à l'école : du texte réticent au texte proliférant », Francis Gossman et Catherine Tauveron (coord.), Repères 19, «Comprendre et interpréter les textes à 1'école », Paris, INRP, pp. 9-38.

- (dir.), (2002) : Lire la littérature à l'école, Pourquoi et comment conduire cet apprentissage spécifique? De la GS au CM, Paris, Hatier Pédagogie.

VergniouX, A. (2003) : L'explication dans les sciences, Bruxelles, de Boeck.

VESLIN, J. (1988) : «Quels textes scientifiques espère-t-on voir les élèves écrire?» quelques exemples de l'utilisation d'une modélisation des textes scientifiques dans un contexte d'évaluation formatrice, Aster 6, Paris, INRP, «Les élèves et l'écriture en sciences », pp. 91-127.

WEISSER, M. (1995) : «L'élève, un interprète professionnel », Aster 21, «Enseignement de la géologie », Paris, INRP, pp. 181-200. 


\section{Annexe}

Schéma de Maxence (volcan effusif)

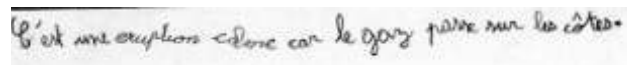

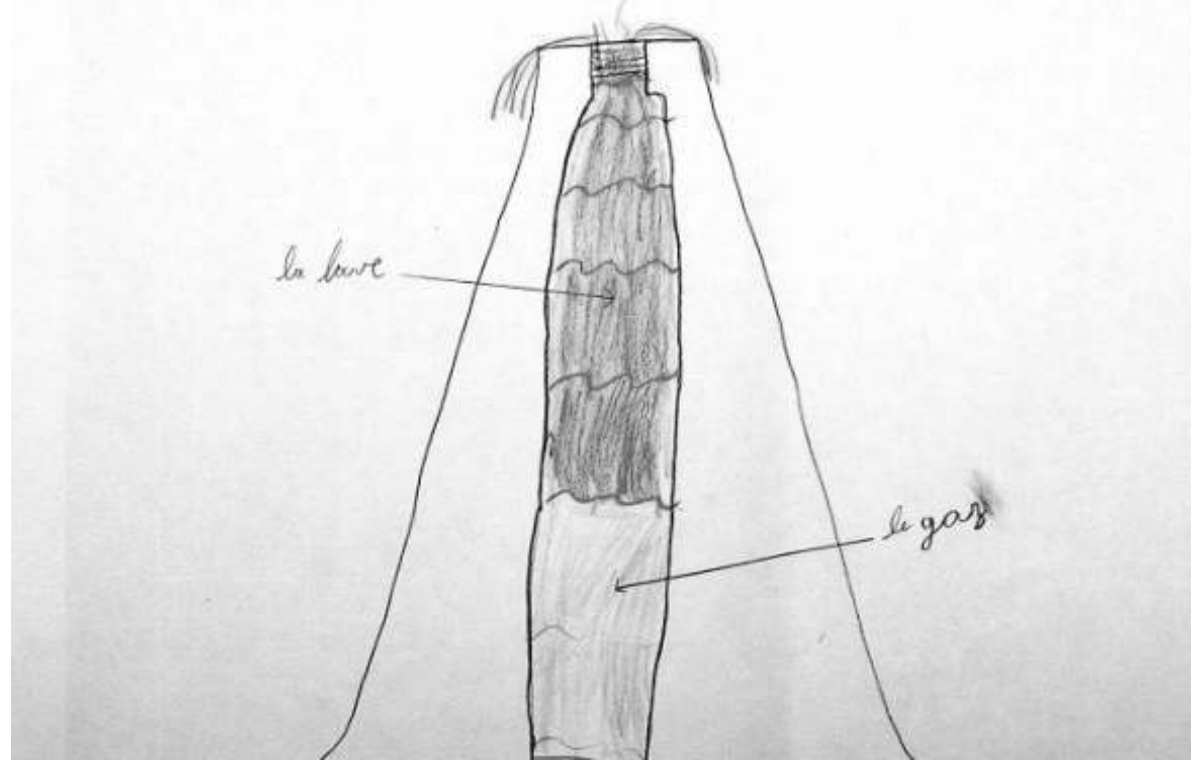

